\title{
Las políticas públicas simbólicas para el buen gobierno y la calidad democrática en la Comunitat Valenciana
}

Symbolic Public Policies of Governance and Democratic

Quality in the Valencian Community

María Ángeles Abellán López • ma.abellan@umh.es

UNIVERSIDAD MIGUEL HERNÁNDEZ DE ELCHE

Gonzalo Pardo Beneyto • gonparbe@gmail.com

UNIVERSIDAD DE ALICANTE

Recibido: 18/12/2016

Aceptado: 16/05/2017

\section{Resumen}

Con la aprobación de la Ley 19/2013, de transparencia, acceso a la información pública y buen gobierno, las Administraciones Públicas subestatales han tenido que implementar una serie de políticas públicas encaminadas a mejorar aspectos como la calidad democrática, la lucha contra la corrupción y la información confiable, entre otros.

Este artículo se propone explorar y describir los primeros pasos que ha dado la Generalitat Valenciana para el aseguramiento de la transparencia y el buen gobierno en su ámbito territorial y, en especial, el estudio del Código de Buen Gobierno, utilizando como marco teórico el neoinstucionalismo.

Palabras clave: Buen Gobierno, Nuevo Institucionalismo, Transparencia, Calidad Democrática, Comunidad Valenciana.

\section{Abstract}

Aproved the Law 19/2013 about transparency, access to public information and good governance, public substate administrations have had to implement a series of public policies to improve aspects such as the quality of democracy, fighting corruption and reliable information, among others.

This paper explores and describes the first steps of Generalitat Valenciana for ensuring transparency and good governance in its territory. Especially, it focuses in the study of the Code of Good Governance. For its purpose, we are going to use a Neoinstitutionalist approach.

Keywords: Good Governance, New Institucionalism, Transparency, Democratic Quality, Valencia Community. 


\section{INTRODUCCIÓN}

Este artículo tiene como objetivo fundamental documentar y describir el nacimiento y los primeros pasos de la Conselleria de Transparencia, Responsabilidad Social, Participación y Cooperación de la Comunitat Valenciana durante su primer año de vida —julio de 2015 a julio de 2016-.

Somos conscientes de las dificultades de plantear un análisis de calado pero parece pertinente - a pesar del poco tiempo transcurrido desde la aprobación de las medidas y la falta de datos empíricos que verifiquen los efectos y la eficacia de todas estas medidas-, referirse al desempeño de unos de los gobiernos del cambio, de la «nueva política», tras las elecciones de 2015, que es el de la Comunitat Valenciana surgido del Pacte del Botànic ${ }^{1}$.

Si bien es cierto que los objetivos de lucha contra la corrupción y el fortalecimiento de la legitimidad del sistema político-administrativo y de sus actores solo se pueden analizar a largo plazo, no por ello es desdeñable conocer de manera aproximativa, en primera instancia, cómo los gobiernos — nuevos y viejos - encaran la difícil temática de la calidad democrática, de la legitimidad y del buen gobierno.

El caso de la Conselleria de Transparencia, Responsabilidad Social, Participación y Cooperación de la Comunitat Valenciana constituye un caso concreto para conocer qué actuaciones se están llevando a cabo en la búsqueda de legitimidad, de adaptación al contexto y de cumplimiento de la legalidad vigente y aunque no sea posible generalizar o inferir resultados sí que consideramos que tiene valor interpretativo.

Nuestra hipótesis inicial es que el origen fundacional de la Conselleria es la necesidad de recuperar la legitimidad perdida y suministrar a esa recuperación una visibilidad pública y notoria puesto que sin legitimidad no puede persistir ningún sistema político. Esta última acepción está íntimamente conectada con aquello que se percibe, así como con los elementos de juicio del individuo y su clasificación valorativa de lo que un acto, una acción, un objeto o un valor es positivo o negativo para sí mismo y la sociedad que lo rodea (Bourdieu, 1997). La legitimación es, de esta forma, un valor percibido que se relaciona directamente con elementos intangibles y socialmente construidos que para las organizaciones son recursos a gestionar y a tener en cuenta (Oliver, 1997).

El marco teórico utilizado es el neoinstitucionalismo y a partir de él, vamos a explorar dos elementos estructurales de la reciente Conselleria: a) la configuración de sus estructuras organizativas y b) los instrumentos de transparencia y buen gobierno promulgados. Am-

\footnotetext{
${ }^{1}$ Los partidos suscribientes son el PSPV, la Coalición Compromís y Podem-Podemos y la denominación está tomada del lugar donde se firmó el acuerdo. 
bos elementos están interconectados puesto que se refieren a la necesidad de dotarse a sí misma de institucionalidad, de estructura, para generar productos legitimadores, es decir, cumplir su mandato democrático.

La disposición del presente trabajo es la siguiente: en primer lugar, fundamentaremos la necesidad de la búsqueda de legitimidad de las instituciones mediante un enfoque neoinstitucionalista y la adopción de instrumentos de transparencia y buen gobierno. Seguidamente, realizaremos una breve aproximación al contexto político de la Comunitat Valenciana y explicaremos la génesis, la organización y los instrumentos de aseguramiento de la Conselleria de Transparencia, Responsabilidad Social, Participación y Cooperación. En este punto nos centraremos en el Código de Buen Gobierno como instrumento más reseñable hasta el momento. Por último, formularemos algunas conclusiones sobre el caso de estudio, con un marcado acento de provisionalidad atendiendo al dinamismo funcional en el que se encuentra inmersa la Conselleria de estudio.

La metodología combinará el análisis documental de los textos legales, la lectura de la bibliografía consultada y el esfuerzo hermenéutico que requiere la interpretación del desarrollo institucional-funcional de la Conselleria, la producción de elementos normativos y su conexión con el marco teórico utilizado en este artículo.

\section{EL NEOINSTITUCIONALISMO Y LA LEGITIMIDAD}

El enfoque neoinstitucionalista es fruto de los cambios acontecidos en el contexto sociopolítico y que acaban por afectar al corpus teórico de la Ciencia Política. Aparece, pues, como una reacción a los estudios que se centraban en el individualismo metodológico ${ }^{2}$ y que, a su vez, se anteponían a los trabajos basados en el institucionalismo clásico que se fundamentaba en una exacerbada juridicidad, en los aspectos formales de las instituciones, en el tratamiento único de una serie de instituciones que conforman el sistema político de un país y en un recurso a la historia poco sofisticado. A partir del artículo de March y Olsen titulado «The New Institutionalism: Organizational Factors in Political Life» las investigaciones vuelven a tener un renovado interés por las instituciones. El enfoque neoinstitucionalista trata de corregir los problemas del viejo institucionalismo, incluir nuevas formas de entender los asuntos públicos y enfatizar la incidencia de los aspectos institucionales en el comportamiento de los actores para explicar el proceso político, desde diferentes perspectivas ${ }^{3}$ (Easton, 1997; Immergut, 1998; Pierson, 2000; Mahoney, 2000; Peters, 2003; Pierson y Skocpol, 2008).

\footnotetext{
${ }^{2}$ En concreto, el neoinstitucionalismo trata de combatir la idea de que a través de los pensamientos y tendencias individuales se pueden tener agregaciones globales que ayuden a establecer pautas globales de comportamiento político. Por lo que se hace referencia al conductismo y a la teoría de la elección racional, enfoques basados en esta idea.

${ }^{3}$ Las diferencias entre los distintos enfoques se explican por el énfasis que se le da a unos elementos respecto a otros. Así por ejemplo, se encuentra el neoinstitucionalismo de la elección racional que se basa en los efectos de las instituciones en la toma de decisiones de los individuos o el neoinstitucionalismo histórico que estudia los procesos históricos con el objetivo de caracterizar los fenómenos políticos actuales (Peters, 2003).
} 
En esta línea, el neoinstitucionalismo sociológico es un enfoque plural ${ }^{4}$ en el que tienen importancia el contexto, la legitimidad y los aspectos organizacionales, así como los culturales. Su base principal se encuentra en los escritos de los clásicos de la sociología como Weber, Durkheim, Selznick o Einsenstadt entre otros (Immergut, 1998; Meyer y Rowan, 1977; Peters, 2003; DiMaggio y Powell, 1991; Selznick, 1996).

Las instituciones realizan cambios en su organización y funcionamiento con el objetivo de incrementar sus recursos y posibilidades de supervivencia, por lo que la utilidad es un elemento que justifica su existencia. El entorno es fundamental, ya que influye en las organizaciones para que éstas lleven a cabo modificaciones en su acción y en sus elementos esenciales. Asimismo, la acción estratégica de los individuos que conforman la organización -y su liderazgo- son factores que marcan las tendencias de implementación de estas constricciones contextuales.

DiMaggio y Powell (1983: 150), identifican tres corrientes que propician un cambio isomórfico: a) el isomorfismo coercitivo que tiene su base en la presión legal y social; b) el isomorfismo mimético en el que se buscan otros modelos que se puedan adaptar a la organización y así hacer frente a la incertidumbre y; c) el isomorfismo normativo que hace referencia a los aspectos profesionales de un trabajo concreto (Deephouse y Suchman, 2008; DiMaggio, 1988; DiMaggio y Powell, 1983; Meyer y Rowan, 1977; Oliver, 1991; Tolbert y Zucker, 1983). Por lo que la legitimidad, no es un recurso, sino que es una característica propia de aquello que está conforme con la cultura preponderante, tiene soporte normativo y cumple las normas y leyes aplicables a la persona, objeto u organización que la ostenta (Scott, 1995: 45).

¿Pero qué es lo que buscan las instituciones del entorno?

Básicamente, buscan generar legitimidad como medida de supervivencia. Weber (2002) entendía la legitimidad como aquella justificación de la que se sirve el poder político para fundamentar su autoridad y de que un determinado orden político sea aceptado pacíficamente. Por su lado, Lipset (2001) la concebía como la capacidad del sistema para mantener la creencia en el funcionamiento de las instituciones políticas como las más adecuadas para la sociedad.

Muchas administraciones públicas han visto cuestionada su legitimidad y sus responsables políticos han adoptado medidas encaminadas a mejorar la transparencia de las instituciones y de la rendición de cuentas. La transparencia contribuye a la apertura de la información y deviene como uno de los principios fundamentales de actuación de los poderes públicos e instituciones representativas. Este principio opera como una condición necesaria

\footnotetext{
${ }^{4}$ Peters (2003) señala que el neoinstitucionalismo sociológico tiene las siguientes ramas, a saber: 1) modelos que siguen la lógica de la ecología de la población y la obtención de recursos; 2) el isomorfismo y los procesos de institucionalización; 3) la sedimentación histórica de cambios y; 4) los arquetipos como elementos de conceptualización.
} 
y general de que la información está disponible para que la ciudadanía pueda utilizarla, observarla y difundirla. La transparencia permite limitar la corrupción, mejorar la capacidad de influencia de la ciudadanía y reforzar el bien común por encima de intereses privados (Belmonte, 2014).

En conexión con la legitimidad se encuentra otro gran concepto como es la rendición de cuentas, accountability, que constituye el fundamento de la democracia representativa. Es incuestionable que existe una pluralidad de partes interesadas en la actividad pública y la rendición de cuentas constituye una búsqueda de legitimidad de las políticas públicas puestas en marcha (Callahan, 2007; Meyer y Rowan, 1977). La rendición de cuentas suscita controversias en el corazón de la teoría democrática en el sentido de conocer si los representantes actúan como delegados (delegates) o como fiduciarios (trustees) (Saward, 2010). La idea que subyace es la necesidad de que las instituciones políticas estén sometidas a controles y los gobernantes tengan la obligación de explicar y justificar cómo han gestionado los asuntos públicos (Schedler, 1999; O’Donnell, 1998, 2003; Morlino, 2009; Mulgan, 2011, 2014).

Para Mulgan (2014), los conceptos de transparencia y rendición de cuentas, forman parte de la expresión popular Open Government pero no constituyen una unidad conceptual. La rendición de cuentas siempre se realiza ex post, una vez que el gestor público o accountor ha realizado las actuaciones que serán objeto del proceso de accountability. Por lo que se refiere a la transparencia, ésta tiene una composición más abierta en cuanto alcance y dirección porque, si bien se ofrece a individuos y organizaciones, no necesariamente ha de estar dirigida de forma específica y explícita a alguien sino que es una condición general de estar disponible esa información para los ciudadanos cuando la necesiten. La mera divulgación de información y datos sin debate tiene poco valor para desarrollar un enfoque de open government (O’Neill, citado por Mulgan 2014:5).

Tanto la transparencia como la accountability son dos conceptos que se relacionan directamente con la evaluación de políticas públicas entendida como el acto de reunir información sobre un programa público y la organización que lo pone en marcha (Harguindeguy, 2013).

La legitimidad requiere la puesta en marcha de políticas simbólicas para transformar la percepción de la ciudadanía, especialmente cuando se relaciona con una situación de degradación institucional (Fountaine, 2015; Suchman, 1995). En este contexto, la políticas simbólicas se refieren a aquéllas políticas que no tienen un resultado material puesto que son intangibles para el público pero que tienen una particular respuesta para la ciudadanía y se relacionan con la generación de estructuras y leyes (Edelman, 1971).

El gran reto de la nueva Conselleria valenciana es precisamente la producción de valores y políticas simbólicas en aras de recuperar la necesaria legitimidad institucional. 


\section{LA COMUNITAT VALENCIANA Y SU ENTORNO}

Las elecciones de mayo de 2015 cerraron un ciclo de dos décadas del Partido Popular como partido hegemónico ${ }^{5}$ (Sartori, 2005). El vuelco producido en la Generalitat Valenciana se explica, entre otros factores, por el desgaste de las formaciones políticas cuando se perpetúan un periodo prolongado de tiempo en el ejercicio de gobierno.

La victoria electoral del Partido Popular en 1995 en la Comunitat Valenciana le permitió acceder a la Generalitat como un reflejo del voto de castigo hacia el PSOE ${ }^{6}$ por lo casos de corrupción (Delgado Sotillos, 1999). Su programa de gobierno estableció, casi desde el principio, la edificación de grandes obras, la organización de multitudinarios eventos, el fomento del turismo de sol y playa, así como la construcción residencial (Roche, 2013; Sanmartín, 2010).

Por otro lado, la integración de Unión Valenciana en el PPCV reforzó los postulados programáticos de un anticatalanismo, que fue utilizado como arma política para consolidarse ante la opinión pública valenciana ${ }^{7}$, con la ayuda de unos medios de comunicación públicos pero sensibles a su línea ideológica y sus reivindicaciones (Xambó, 2010).

Relacionado directamente con el deseo de pervivencia que toda formación política tiene en un sistema de partidos imbuido por la cartelización (Katz y Mair, 1995), se produjo una simbiosis entre las instituciones y los recursos públicos que degeneró en una confusión - por parte de algunos dirigentes y cargos públicos del Partido Popular- entre lo público, el partido y el lucro individual. En un escenario como el descrito, se fraguaron ciertas redes informales basadas en la confianza y en la lealtad partidista que se han visto implicadas en casos de corrupción (Della Porta, 2001) como, por ejemplo, el Caso Terra Mítica, la Trama Gürtel, el Caso Cooperación o el Caso Taula, entre otros.

Otro elemento a tener en cuenta es la evidencia de los datos publicados por el Ministerio de Hacienda y Función Pública y en los que consta que la Comunitat Valenciana tiene un problema de infrafinanciación respecto a otros territorios ${ }^{8}$. El problema de la financiación autonómica ha pasado a priorizar la agenda pública en la Comunitat Valenciana puesto

\footnotetext{
5 Antes, el PSPV-PSOE había puestos los cimientos de las instituciones autonómicas valencianas, durante el periodo 1982-1995.

${ }^{6}$ Dicho cambio en las preferencias de voto se explica por el carácter de segundo orden de estas elecciones (Reif y Schmitt, 1980).

${ }^{7}$ Por un lado, los autonomistas reivindicaron la cooficialidad de la lengua catalana, la unidad lingüística, una bandera sin una banda azul y el escudo de la Generalitat, así como la denominación de País Valencià, y se alinearon con posiciones que simpatizaban con el catalanismo. Enfrentados a ellos, se encontraba un sector conservador —el blaverismo - que acusaba a los autonomistas de "antivalencianos» y asumía el tradicionalismo basado en el antiguo Reino de Valencia, en la bandera coronada con franja azul y afirmaba el valenciano como lengua propia y distinta del catalán (Sanz Díez, 1982; Sanz Díez y Felip i Sardà, 2006).

8 Véase Informe sobre los Presupuestos de las Administraciones Públicas para 2017: Comunitat Valenciana elaborado por la Autoridad Independiente de Responsabilidad Fiscal en:

http://www.airef.es/system/assets/archives/000/002/028/original/2017_04_26_Informe_ppto2017_abril_CVA. pdf?1493203109
} 
que se parte de la consideración que ésta contribuye a la recaudación de las arcas estatales pero que en la redistribución obtiene una renta por debajo de la media. Este panorama ha afectado a la calidad de los servicios públicos alimentando una crisis de eficacia y de legitimidad en las políticas públicas ${ }^{9}$. Todos estos factores han facilitado, en mayor o menor medida, que se produzca un deterioro de las instituciones públicas en cuanto a la percepción de la ciudadanía.

Como veremos en el siguiente epígrafe, a partir del cambio de gobierno gestado en las elecciones a Cortes Valencianas de mayo de 2015, los nuevos gobernantes han puesto en marcha una serie de medidas para revertir las situaciones y percepciones mencionadas con el gran reto de producir incrementos en la legitimidad de las instituciones de autogobierno.

\section{LA GÉNESIS Y LA ESTRUCTURACIÓN DE LA CONSELLERIA DE TRANSPARENCIA, RESPONSABILIDAD SOCIAL, PARTICIPACIÓN Y COOPERACIÓN}

La estructura básica y las competencias de la Conselleria de Transparencia, Responsabilidad Social, Participación y Cooperación son establecidas por el Decreto 103/2015, por el que se establece la estructura orgánica básica de la Presidencia y de las conselleries de la Generalitat $^{10}$. Su configuración estructural y competencial está regulada, con más detalle, en el Reglamento Orgánico y Funcional de la Conselleria de Transparencia, Responsabilidad Social, Participación y Cooperación ${ }^{11}$. Concretamente, esta Conselleria tiene atribuido el mandato legal de diseñar las políticas públicas en las siguientes materias: a) la transparencia de la actividad pública; b) la participación de la sociedad civil y ciudadana; c) la responsabilidad social, d) el fomento del autogobierno y el desarrollo estatutario; e) la cooperación al desarrollo y la solidaridad. La creación de esta división organizativa por parte de la Presidencia de la Generalitat obedece, a nuestro juicio, a dos razones fundamentales: la primera de ellas es hacer frente a los compromisos del Acuerdo del Botánico ${ }^{12}$ y en segundo lugar, a la necesidad de legitimar el nuevo Consell de la Generalitat surgido de las elecciones de mayo de 2015, dotando de un mayor protagonismo las materias relacionadas con la mejora del Buen Gobierno y la Calidad Democrática, además de la mejora del autogobierno valenciano.

\footnotetext{
${ }^{9}$ Algunos ejemplos de este hecho son los retrasos producidos en las subvenciones, el aumento de la ratio en los colegios o la falta de inversiones prioritarias en la construcción de hospitales o en la modernización del transporte público.

${ }^{10}$ En base a lo dispuesto en la Ley 5/1983, de 30 de diciembre, del Consell.

11 Decreto 160/2015, de 18 de septiembre, del Consell, por el que aprueba el Reglamento Orgánico y Funcional de la Conselleria de Transparencia, Responsabilidad Social, Participación y Cooperación.

${ }^{12}$ El Acuerdo del Botánico, conocido como el Pacte del Botànic, es el documento que contiene todos aquellos compromisos asumidos por los partidos que forman parte del gobierno (PSPV-PSOE y Compromís) y Podemos CV como socio parlamentario. En concreto se hace referencia al punto dos del acuerdo que trata la Regeneración democrática y lucha contra la corrupción, aunque sus valores se encuentran presentes durante todo el documento.
} 
Para la consecución de estos objetivos, se dispone de una estructura weberiana clásica en la que se prima la jerarquía y la especialización sobre otro tipo de valores; por lo que su estructura se configura en base a grupos de tareas en un área determinada. Se puede afirmar, en este aspecto, que se sigue un modelo ministerial inspirado en la Administración Central española. El fundamento del modelo se debe a la búsqueda de legitimidad respecto al resto de Conselleries y Sector Público valenciano al generar estructuras similares a las que ya existen (Leblebici et al., 1991).

No obstante, este se combina con estructuras propias en cada Conselleria que forma el gobierno de la Generalitat y que son las encargadas de conectar la Conselleria de Transparencia con el resto. Estas unidades administrativas tienen distintos encajes variando su rango. En algunas de ellas se configura en la misma subsecretaria ${ }^{13}$, en la subsecretaria general administrativa ${ }^{14}$, en algunos servicios ${ }^{15} \mathrm{u}$ en otras dependencias ${ }^{16}$.

Desde un punto de vista interno, la Conselleria asegura que se cumplen los parámetros dispuestos sobre transparencia y otros valores normativos a través de la Inspección General de la Generalitat.

Tabla 1. Estructura del diseño, implementación, evaluación y control de la transparencia y el buen gobierno

\begin{tabular}{|l|l|}
\hline \multicolumn{1}{|c|}{$\begin{array}{c}\text { Conformador de las Políticas de Transparencia y } \\
\text { Buen Gobierno }\end{array}$} & \multicolumn{1}{c|}{$\begin{array}{c}\text { Sistema de soporte y asegurador interno de la } \\
\text { transparencia y el Buen Gobierno }\end{array}$} \\
\hline $\begin{array}{l}\text { Conselleria de Transparencia, Responsabilidad } \\
\text { Social, Participación y Cooperación }\end{array}$ & $\begin{array}{l}\text { Subsecretarias, subsecretarias generales } \\
\text { administrativas, servicio, otras dependencias }\end{array}$ \\
\hline Organismo de Control Independiente & Organismo de Control Interno \\
\hline $\begin{array}{l}\text { Consejo de Transparencia, Acceso a la } \\
\text { Información Pública y buen Gobierno }\end{array}$ & Inspección General de Servicios \\
\hline
\end{tabular}

Fuente: Elaboración propia a partir de datos de Conselleria de Transparència (2016).

Otro de los elementos importantes dedicados a la transparencia es el Consejo de Transparencia, Acceso a la Información Pública y Buen Gobierno. Este organismo es el encargado

\footnotetext{
${ }^{13}$ La subsecretaría se encarga de la transparencia directamente en las siguientes Consellerias: Presidencia; Vicepresidencia de Igualdad/ Conselleria de Igualdad y Políticas Inclusivas; Conselleria de Educación, Investigación, Cultura y Deporte, Transparencia Responsabilidad Social, Participación y Cooperación.

${ }^{14}$ La subsecretaría general administrativa gestiona los asuntos que tienen que ver con la transparencia en las siguientes Consellerias: Conselleria de Justicia, Administración Pública, Reformas Democráticas y Libertades Públicas; Conselleria de Hacienda y Modelo Económico; Conselleria de Sanidad Universal y Salud Pública

${ }^{15}$ En la Conselleria de Economía Sostenible, Sectores Productivos, Comercio y Trabajo es el servicio de Modernización y Simplificación Administrativa adscrito a la subsecretaría el que se encarga de los temas de transparencia. En la Conselleria de Vivienda, Obras Públicas y Vertebración del Territorio depende del Servicio de Organización y Técnicas de Gestión dependiente de la Subsecretaría General Administrativa.

${ }^{16}$ En la Conselleria de Agricultura, Medio Ambiente, Cambio Climático y Desarrollo Rural es la Subdirección General del Gabinete Técnico la que se encarga de los temas de transparencia.
} 
de garantizar el acceso a la ciudadanía a la información, controlar que se publica información de relevancia pública y observar el cumplimiento de las medidas de Buen Gobierno. Desde el punto de vista funcional, es independiente de cualquier Administración valenciana, aunque está adscrita, desde un punto de vista estructural, a la Conselleria de Transparencia, Responsabilidad Social, Participación y Cooperación.

\subsection{Los instrumentos de aseguramiento del buen gobierno}

Los primeros pasos de la Conselleria de Transparencia, Responsabilidad Social, Participación y Cooperación han ido encaminados a tejer una serie de relaciones entre distintas organizaciones públicas y privadas, como sucede en el marco de Gobernanza en el que nos hallamos inmersos. Buen ejemplo de ello, es la firma de sendos convenios con las universidades públicas valencianas y Transparencia Internacional para el fomento de la transparencia y el buen gobierno; la realización de reuniones de trabajo con distintos interlocutores provenientes de la sociedad civil en las competencias en la que Conselleria es competente y con entidades locales, otras comunidades autónomas y organizaciones públicas estatales competentes en la materia (Conselleria de Transparència, 2016).

La Conselleria, además, ha potenciado las relaciones públicas, participando en jornadas, seminarios, conferencias y actos institucionales variados. También, se ha tomado la decisión de estudiar todas aquellas medidas que se pusieron en marcha en la anterior legislatura y realizar cambios parciales en su seno. Este es el caso, por ejemplo, de la promulgación de la Ley de Cuentas Abiertas de la Generalitat Valenciana y el cambio del Portal de Transparencia de la Generalitat Valenciana para hacerlo más accesible e intuitivo. También, se ha aprobado un Código de Buen Gobierno que será estudiado en el siguiente subepígrafe.

Tal como se puede comprobar en la Tabla 2, desde julio de 2016, esta Conselleria ha ido realizando una serie de contribuciones a la mejora de la transparencia, la participación y la colaboración y, en definitiva, el buen gobierno que se ampliará en los próximos tiempos.

Uno de los desarrollos normativos en los que se está trabajando actualmente es la Ley de Responsabilidad Social que tiene como objetivo fomentar las buenas prácticas que tengan en cuenta aspectos como la ética, el medio ambiente, la igualdad y el bienestar de los trabajadores, entre otros y que afectará tanto al sector público y privado. Asimismo, se está fraguando una mejora del funcionamiento de la Ley 2/2015 con la promulgación de un Reglamento que defina los aspectos capitales de la transparencia. Por último, se está avanzando en la regulación y puesta en marcha del Consejo de Participación Ciudadana de la Comunitat Valenciana. 
Las políticas públicas simbólicas para el... | María Ángeles Abellán López, Gonzalo Pardo Beneyto

Tabla 2. Compromisos adoptados por la Conselleria de Transparencia, Responsabilidad Social, Participación y Cooperación hasta el 1 de julio de 2016

\begin{tabular}{|l|l|}
\hline $\begin{array}{l}\text { Decreto 160/2015, de } 18 \text { de septiembre, del Consell, por el que aprueba } \\
\text { el Reglamento Orgánico y Funcional de la Conselleria de Transparencia, } \\
\text { Responsabilidad Social, Participación y Cooperación. }\end{array}$ & Aprobado \\
\hline Cambio de Portal de Transparencia & Finalizado \\
\hline $\begin{array}{l}\text { Generalitat Valenciana. [2016/3260] LEY 5/2016, de 6 de mayo, de Cuentas } \\
\text { Abiertas para la Generalitat Valenciana [Presidencia] }\end{array}$ & Aprobado \\
\hline $\begin{array}{l}\text { Decreto 56/2016, del Consell, de 6 de mayo, por el que se aprueba el Código } \\
\text { de Buen Gobierno de la Generalitat. }\end{array}$ & Aprobado \\
\hline $\begin{array}{l}\text { Decreto que regula y desarrolla la Ley 2/2015 respecto a la aplicación } \\
\text { de la transparencia para resolver los aspectos administrativos y de } \\
\text { funcionamiento }\end{array}$ & En proceso \\
\hline $\begin{array}{l}\text { Proyecto de Ley Valenciana de Fomento de la Responsabilidad Social para } \\
\text { incorporar a la contratación pública e impulsarla en el sector privado }\end{array}$ & En proceso \\
\hline $\begin{array}{l}\text { Decreto por el que se regula el Consejo de Participación Ciudadana de la } \\
\text { Comunitat Valenciana }\end{array}$ & En proceso \\
\hline Proyecto de Ley de Regulación de los Grupos de Interés y Presión (Lobbies) & Próximo desarrollo \\
\hline $\begin{array}{l}\text { Articulación sobre un espacio de colaboración para la prevención y lucha } \\
\text { contra la corrupción }\end{array}$ & Próximo desarrollo \\
\hline $\begin{array}{l}\text { Diseño de un sistema estable de asesoramiento y formación para el tejido } \\
\text { asociativo. Elaboración de la propuesta de Ley de Participación y Soporte del } \\
\text { Asociacionismo }\end{array}$ & Próximo desarrollo \\
\hline
\end{tabular}

Fuente: Elaboración propia a partir de Presidencia (2016).

Como objetivos a largo plazo, se encuentra la regulación de los lobbies y grupos de interés, la articulación de nuevos espacios de colaboración para la prevención y lucha contra la corrupción; así como la puesta en marcha de un servicio para el asesoramiento y la formación de los miembros del tejido asociativo.

\subsection{El Decreto 56/2016, del Consell, de 6 de mayo, por el que se aprueba el Código de Buen Gobierno de la Generalitat}

Una de las medidas más destacables puestas en marcha hasta el momento por la Conselleria estudiada es la promulgación de un Código de Buen Gobierno de la Generalitat. Su objetivo es buscar legitimar la acción de aquellos que lo adoptan a través de una obligación moral hacia una serie de partes interesadas. Al final, el Código de Buen Gobierno en los países de tradición francesa trata de aunar el interés propio con los valores de la sociedad en la que está establecida una sociedad mediante instrumentos normativos laxos y que complementan a la ley (Long y Driscoll, 2007; Conselleria de Transparencia, 2016; Zattoni y Cuomo, 2008). 
Este documento normativo es obligatorio para los órganos superiores, el nivel directivo de Consellerias y Organismos pertenecientes al Sector Público Valenciano ${ }^{17}$. Este Código también se aplicará, de forma voluntaria, a los miembros de las Corts, el Síndic de Greuges, la Sindicatura de Cuentas, el Consejo Valenciano de Cultura, la Academia Valenciana de la Lengua, el Comité Económico y Social, el Consejo Jurídico-Consultivo y cualquier otra institución análoga. Asimismo, podrán adoptarlo los miembros de las corporaciones locales y su personal directivo y las Universidades Públicas.

Las medidas morales y éticas de este documento se pueden dividir en tres categorías:

1. La sujeción a los principios legales que rigen en la sociedad valenciana, en la que se contemplan los aspectos normativos y de derechos que se encuentran en la Constitución, el Estatuto de Autonomía, la Carta de Derechos Fundamentales de la Unión Europea y la Declaración Universal de los Derechos Humanos y resto de documentos legales, internacionales o nacionales, que tienen que ver con el reconocimiento subjetivo de derechos y libertades públicas. Asimismo, se centran en el respeto de la igualdad en todo su significado, la defensa de la lengua propia y la condena expresa a la violencia, entre otros aspectos. Como se puede observar, el Código incide en aspectos normativos que son deseables en la sociedad en la que se intrican sus firmantes y el puesto que ocupan.

2. La integridad y la ejemplaridad de la que todo servidor público debe hacer gala. En este punto se incide en el comportamiento de los cargos públicos y como deben ser un ejemplo para el resto de la sociedad, buscando la imparcialidad, la racionalidad de las decisiones y la confidencialidad. De la misma forma, el Código aboga por fomentar el uso de la comunicación como servicio público y, así, obliga a los firmantes a no usar los medios de comunicación con fines partidistas y propagandistas. Es por esto que se regula el uso de la imagen pública de las instituciones en las redes sociales, el de la publicidad institucional y, en definitiva, el empleo del dinero público en acciones comunicativas. Por último, se promueve la responsabilidad y la rendición de cuentas como valores para establecer una relación de control entre la ciudadanía, las instituciones de control y el propio firmante.

3. La austeridad en el uso de recursos públicos en el ejercicio de sus responsabilidades constituye uno de los ejes discursivos clave. Este grupo de artículos se vertebra a través de la idea de que los recursos públicos son escasos por lo que su uso debe ser justificado para atender a los deberes que el cargo impone a su propietario. En este punto, el Código entra a regular el uso del coche oficial, las compras, el régimen de regalos y la prohibición del uso de tarjetas públicas, entre otros.

4. Por último, se hace una mención expresa al impulso del Gobierno Abierto y a la buena Administración. Para ello, se fija la información personal del cargo a publicar en la web del organismo, también se regulan las relaciones con la ciudadanía, las Cortes y el

\footnotetext{
${ }^{17}$ Según el Artículo 2.3 de la Ley 1/2015, de 6 de febrero, de la Generalitat de Hacienda Pública, del Sector Público Instrumental y de Subvenciones el sector público está compuesto por: los organismos autónomos, las entidades públicas empresariales, otras entidades de Derecho Público distintas de las anteriores, las Sociedades mercantiles, las Fundaciones y los Consorcios en los que sus actos estén sujetos de forma directa o indirecta a la Generalitat.
} 
personal administrativo de la institución. En definitiva, se pretende que los cargos superiores y directivos sean el motor de la regeneración democrática en la Generalitat mediante el cumplimiento de este Código de Buen Gobierno.

En otro orden de cosas, también, se regulan de forma parsimoniosa los aspectos que tienen que ver con las medidas coercitivas y se incluye la regulación del Registro de actividades y bienes públicos para altos cargos de la Generalitat.

\section{CONCLUSIONES}

La Conselleria de Transparencia es una consecuencia práctica del trabajo realizado por el actual gobierno surgido del Pacte del Botànic. Su génesis responde al objetivo de restaurar legitimidad a través de políticas simbólicas y normativas. Su ámbito competencial, además, va más allá, poniendo en marcha medidas relacionadas con la participación, el buen gobierno, la responsabilidad social, el fomento del autogobierno y la cooperación al desarrollo.

El Código de Buen Gobierno de la Generalitat es un nuevo instrumento con potencial para asegurar la calidad democrática de las instituciones autonómicas y que comporta una visión menos tecnocrática y juridicista que la Ley de Transparencia y Buen Gobierno estatal. Como instrumento normativo no tiene repercusiones jurídicas aunque sus preceptos tienen importancia desde un punto de vista prescriptivo-normativo, por lo que pueden orientar las conductas que son deseables y valiosas políticamente. Esta idea apoya nuestra hipótesis inicial y es que la Conselleria busca a través de sus políticas simbólicas recuperar la legitimidad y potenciar la visibilidad pública de la Generalitat.

Este artículo, como ya se ha señalado, tiene un carácter exploratorio dado que no ha transcurrido un plazo temporal suficiente que ofrezca una perspectiva global y las conclusiones, por tanto, son provisionales. Sin embargo, pensamos que el carácter exploratorio y la provisionalidad no han de constituir un hándicap para reflexionar sobre las primeras acciones desarrolladas por la Conselleria para mejorar la calidad democrática. No se debe perder de vista la necesidad de conocer los principales instrumentos puestos en marcha y las motivaciones que los mueven y los promueven.

Por otro lado, ha de considerarse que las políticas públicas desarrolladas experimentarán modificaciones para aumentar su rendimiento y que esta situación abrirá nuevas vías de investigación, a partir del análisis comparado de las distintas políticas de mejora de la legitimidad de otros territorios.

Finalmente, recomendamos a la Conselleria de Transparencia la conveniencia de generar indicadores de medición y de evaluar los principales efectos de las políticas públicas simbólicas implementadas, usando los medios y la metodología disponibles en el Plan de la Inspección de Servicios de la Generalitat Valenciana. 


\section{FUENTES LEGALES CONSULTADAS}

BOE (2013). Ley 19/2013, de 9 de diciembre, de transparencia, acceso a la información pública y buen gobierno (Texto Consolidado) [PDF]. [Fecha de consulta: 14/09/2016]. $<$ https://www.boe.es/buscar/act.php?id=BOE-A-2013-12887>

BOE (2016). Ley 2/2015, de 2 de abril, de Transparencia, Buen Gobierno y Participación Ciudadana de la Comunitat Valenciana (Texto Consolidado) [PDF]. [Fecha de consulta: 14/09/2016]. <https://boe.es/buscar/pdf/2015/BOE-A-2015-4547-consolidado.pdf>

Comunitat Valenciana. Decreto 103/2015, de 7 de julio, del Consell, por el que establece la estructura orgánica básica de la Presidencia y de las consellerias de la Generalitat. Diario Oficial de la Comunitat Valenciana, 08 de julio de 2015 (7566), 21644-21656.

Comunitat Valenciana. Decreto 160/2015, de 18 de septiembre, del Consell, por el que aprueba el Reglamento Orgánico y Funcional de la Conselleria de Transparencia, Responsabilidad Social, Participación y Cooperación. Diario Oficial de la Comunitat Valenciana, 22 de septiembre de 2015 (7620), 25800-25814.

Comunitat Valenciana. Ley 5/2016, de 6 de mayo, de cuentas abiertas para la Generalitat Valenciana. Boletín Oficial del Estado, 01 de junio de 2016 (132), 35973-35978.

Comunitat Valenciana. Decreto 56/2016, del Consell, de 6 de mayo, por el que se aprueba el Código de Buen Gobierno de la Generalitat. Diario Oficial de la Comunitat Valenciana, 13 de mayo de 2016 (7781), 11415-11438.

DOCV (2013). Ley 5/1983, de 30 de diciembre, del Consell. Legislación Consolidada [PDF]. [Fecha de consulta: 14/09/2016]. < http://www.docv.gva.es/rlgv/es/almacenes/indices/ indice_cronologico/signatura/198300501/>

\section{BIBLIOGRAFÍA}

Abellán, M.A. (2014). Republicanismo Contemporáneo y Representación. La ineludible convergencia. Revista Política, vol. 1, n. ${ }^{\circ}$ 52, pp. 195-210.

Belmonte, I. (2014). La transparencia, el buen gobierno y la rendición de cuentas. En J.M. Canales y J.J. Sanmartín (eds.). Introducción a la Ciencia Política (pp. 347-360). Madrid: Universitas.

Bourdieu, P. (1997). Razones prácticas. Sobre la teoría de la acción. Barcelona: Editorial Anagrama.

Callahan, K. (2007). Elements of Effective Governance Measurement, Accountability and Participation. Boca Raton: Taylor Francis Group. 
Conselleria de Transparència (2016). Memòria juliol-desembre 2015 [PDF]. [Fecha de consulta: 14/09/2016]. <http://www.transparencia.gva.es/documents/ 162282364/162406386/Memoria+2015+Conselleria+de+Transparencia, $\% 20$ Responsabilidad+Social,\%20Participaci\%C3\%B3n+y+Cooperaci\%C3\%B3n/bfee172e4677-4548-9a76-922b11a7aa9f>

Deephouse, D.L. y Suchman, M. (2008). Legitimacy in Organizational Institutionalism. En R. Greenwood, C. Oliver, R. Suddaby, K. Sahlin (eds.). The SAGE handbook of Organizational Institutionalism (pp. 49-77). Thousand Oaks: SAGE.

Delgado Sotillos, I. (1999). Resultados electorales y orientación del voto en los comicios municipales de 1995. Reis. Revista Española de Investigaciones Sociológicas, n. ${ }^{\circ}$ 86, pp. 247-273.

Della Porta, D. (2001). Los actores de la corrupción: políticos de negocios en Italia. Gestión y Análisis de Políticas Públicas, n. ${ }^{\circ}$ 21, pp. 23-34.

DiMaggio, P (1988). Interest and Agency in Institutional Theory. En L. G. Zucker (ed.). Institutional patterns and organizations: Culture and environment (pp. 3-21). Cambridge, MA: Ballinger.

DiMaggio, P. y Powell, W.W. (1983). The iron cage revisited: institutional isomorphism and collective rationality in Organizational Fields. American Sociological Review, vol. 2, n. ${ }^{\circ} 48$, pp. $147-160$.

DiMaggio, P. y Powell, W.W. (1991). The new institutionalism in organizational analysis. Chicago: The University of Chicago Press.

Easton, D. (1997). The future of the post-behavioral phase in Political Science. En K.R. Monroe (ed.). Contemporary empirical political theory (pp. 13-46). Berkley: University of California Press.

Edelman, M. (1971). Politics as symbolic action. Mass Arousal and quiescence. Nueva York: Academic Press.

Fountaine, G. (2015). El análisis de políticas públicas. Conceptos, teorías y métodos. Barcelona: Anthropos.

Harguindeguy, J.B. (2015). Análisis de políticas públicas. Madrid: Tecnos.

Immergut, E. (1998). The theoretical core of the new institutionalism. Politics and Society, vol. 1 , n. ${ }^{\circ} 26$, pp. 5-34.

Katz, R. y Mair, P. (1995). Changing models of party organization and party democracy. Party Politics, vol. 1, n. ${ }^{0}$ 1, pp. 5-28. 
Leblebici, H., Salancik, G.R., Copay, A. y King, T. (1991). Institutional Change and the Transformation of Interorganizational fields. Administrative Science Quarterly, vol. 3, n. ${ }^{\circ} 36$, pp. 333-363.

Lipset, S.M. (2001). Algunos requisitos sociales de la democracia: desarrollo económico y legitimidad política. En A. Batlle (ed.). Diez textos básicos de Ciencia Política (pp. 113150). Barcelona: Ariel.

Long, B. y Driscoll, C. (2007). Codes of ethics and the pursuit of organizational legitimacy: Theoretical and empirical contributions. Journal of Business Ethics, vol. 2, n. ${ }^{\circ}$ 77, pp. 173-189.

Mahoney, J. (2000). Path Dependence in Historical Sociology. Theory and Society, vol. 4, n. ${ }^{\circ} 29$, pp. 507-548.

Meyer, J.W. y Rowan, B. (1977). Instituzionalized Organizations: formal structure as myth and ceremony. American Journal of Sociology, vol 2, n. ${ }^{\circ}$ 83, pp. 340-363.

Morlino, L. (2009). Democracia y democratizaciones. Madrid: CIS.

Mulgan, R. (2011). Accountability. En B. Berg-Schlosser y L. Morlino (Eds.). International Encyclopedia of Political Science 1 (pp. 1-13). Thousand Oaks: Sage.

Mulgan, R. (2014). Making open Government Work. Nueva York: Palgrave Mcmillan.

O’Donnell, G. (1998). Accountability horizontal. Estudios Políticos, vol. 4, n. ํ 19, pp. 9-46.

O’Donnell, G. (2003). Horizontal Accountability: The Legal Institutionalization of Mistrust. En S. Mainwaring y C. Welna (eds.). Democratic Accountability in Latin America (pp. 34-54). Nueva York: Oxford University Press.

Oliver, C. (1991). Strategic responses to institutional processes. Academy of Management Review, vol. 1, n. ${ }^{\circ}$ 16, pp. 145-179.

Oliver, C. (1997). Sustainable Competitive Advantage: Combining Institutional and Resource-Based Views. Strategic Management Journal, vol. 18, n. ${ }^{\circ}$ 9, pp. 697-713.

Peters, G. (2003). Institutional Theory in Political Science. The 'New Institutionalism. London: Pinter.

Pierson, P. (2000). Increasing Returns, Path Dependence and the Study of Politics. American Political Science Review, vol 2, n. ${ }^{\circ}$ 94, pp. 251-267.

Pierson, P. y Skocpol, T. (2008). El institucionalismo histórico en la Ciencia Política Contemporánea. Revista uruguaya de Ciencia Política, vol. 1, n. ${ }^{\circ}$ 17, pp. 7-38.

Presidencia (2016). Seminari de govern. Estiu 2016 [PDF]. [Fecha de consulta: 14/09/2016]. <http://www.gvaoberta.gva.es/documents/7843050/163487840/Compromisos+Torrevi eja+Valenci\%C3\%A0.pdf/c3350c9e-720b-46f8-ace9-e8352697ccb5?platform=hootsuite > 
Reiff, K. y Schmitt, H. (1980). Nine second-order national elections -a conceptual framework for the analysis of European election results. European Journal of Political Research, vol. 8, n. ${ }^{\circ}$, pp. 3-44.

Roche, J.A. (2013). La cultura valenciana, entre la realidad y la ficción. En J.M. Canales, y M. Menéndez, (eds.). El sistema político y administrativo valenciano (pp. 20-41). Valencia: Tirant Lo Blanch.

Sanmartín, J.J. (2010). La Comunitat Valenciana en el segle XхI. Reptes $i$ solucions. Sant Vicent del Raspeig: ECU.

Sanz Díez, J. (1982). La cara secreta de la política valenciana. De la predemocracia al Estatuto de Benicassim. Valencia: F. Torres.

Sanz Díez, B. y Felip i Sardà, J. M. (2006). La construcción política de la Comunitat Valenciana, 1962-1982. Valencia: Alfons El Magnànim.

Sartori, G. (2005). Partidos y sistemas de partidos. Marco para un análisis. Madrid: Alianza.

Saward M. (2003). «Representative and Direct Democracy» in Rolan Axtmann (ed.) Understanting Democratic Politics. An Introduction. London: Sage Publications.

Schedler A. (1999). Conceptualizing Accountability. En A. Schedler, L. Diamond \& M.F. Plattner, The Self-Restraining State: Power and Accountability in New Democracies (pp. 13-28). London, Lynne Rienner Publishers.

Scott, R. (1995). Institutions and organizations. Newsbury Park: SAGE Publications.

Selznick, P. (1996). Institutionalism «Old» and «New». Administrative Science Quarterly, vol. 2 , n. ${ }^{\circ} 41$, pp. $270-277$.

Suchman, M. (1995). Managing Legitimacy: Strategic and Institutional Approaches. The Academy of Management Review, vol. 20, n. ${ }^{\circ}$ 3, pp. 571-610.

Tolbert, P. y Zucker, L. (1983). Institutional Sources of Change in the Formal Structure of Organizations: The Diffusion of Civil Service Reform, 1880-1935. Administrative Science Quarterly, vol 1, n. ${ }^{\circ}$ 28, pp. 22-39.

Xambó, R. (2010). Els mitjans de comunicación al País Valencià. Arxius de Ciències Socials, n. ${ }^{\circ} 23$, pp. 3-16.

Weber, M. (2002). Economía y Sociedad. México D.F: Fondo de Cultura Económica.

Zattoni, A. y Cuomo, F. (2008). Why adopt codes of good governance? A comparison of institutional and efficiency perspectives. Corporate Governance, n. ${ }^{\circ}$ 16, pp. 1-15. 